\title{
Spectrum-Occupancy Aware Cooperative Spectrum Sensing Using Adaptive Detection
}

DOI:

10.1109/JSYST.2019.2922773

\section{Document Version}

Accepted author manuscript

Link to publication record in Manchester Research Explorer

\section{Citation for published version (APA):}

Al-Jarrah, M., Al-Dweik, A., Ikki, S., \& Alsusa, E. (2020). Spectrum-Occupancy Aware Cooperative Spectrum Sensing Using Adaptive Detection. IEEE Systems Journal, 14(2), 2225 - 2236.

https://doi.org/10.1109/JSYST.2019.2922773

\section{Published in:}

IEEE Systems Journal

\section{Citing this paper}

Please note that where the full-text provided on Manchester Research Explorer is the Author Accepted Manuscript or Proof version this may differ from the final Published version. If citing, it is advised that you check and use the publisher's definitive version.

\section{General rights}

Copyright and moral rights for the publications made accessible in the Research Explorer are retained by the authors and/or other copyright owners and it is a condition of accessing publications that users recognise and abide by the legal requirements associated with these rights.

\section{Takedown policy}

If you believe that this document breaches copyright please refer to the University of Manchester's Takedown Procedures [http://man.ac.uk/04Y6Bo] or contact uml.scholarlycommunications@manchester.ac.uk providing relevant details, so we can investigate your claim.

\section{OPEN ACCESS}




\begin{abstract}
In this paper, an efficient adaptive detection scheme is proposed for cognitive radio networks where multiple secondary users (SUs) cooperate to identify idle spectrum bands. Each SU generates a binary local decision which is then transmitted either directly or via the assistance of intermediate relays to a cognitive radio base-station (CRBS), where the global decision is made. The fusion process at the CRBS is performed using the local binary decisions made by the individual SUs. The local binary decisions at the relays and CRBS are regenerated based on dynamic thresholds, which are chosen to minimize the probability of spectrum sensing error (PSSE) by considering the imbalanced nature of the spectrum occupancy, as well as the reliability of the decisions made by the SUs. The PSSE performance is derived where a closed-form analytical expression is obtained. The analytical results corroborated by Monte Carlo simulation show that using adaptive detection can reduce the PSSE significantly as compared to the conventional detection approach.
\end{abstract}

Index Terms-Decision fusion, relays, distributed spectrum sensing, cognitive radio network, decode-and-forward, cooperative communications.

\section{INTRODUCTION}

Increasing the spectral efficiency of wireless networks is currently one of the most predominantly pressing requirements for the next generation of wireless communications systems. Although massive research efforts have been devoted to develop spectrally-efficient communication systems, the total bandwidth requirements per user are drastically increasing [1]. Such increase is due to the proliferation of spectrum-hungry high data rate applications, such as video communications and online gaming [2]. As such, the data traffic per smartphone per month in North America has increased from 3.6 GB in 2015 to $5.1 \mathrm{~GB}$ in 2016, and it is expected to exceed 25 GB in 2022, an increase of about 5-fold [3]. The demand for spectrally-efficient systems is consequently expected to sustain its momentum.

Cognitive radio networks (CRNs) have been recently proposed as an effective solution to alleviate the spectrum scarcity

M. Al-Jarrah and A. Al-Dweik are with the Department of Electrical and Computer Engineering, Khalifa Univesity of Science and Technology, Abu Dhabi, UAE. (e-mail: \{mohammad.aljarrah, arafat.dweik\}@ku.ac.ae).

A. Al-Dweik is also with the Department of Electrical and Computer Engineering, Western University, London, ON, Canada.

$\mathrm{S}$. Ikki is with the Electrical Engineering Department, Lakehead University, Thunder Bay, ON P7B 5E1, Canada. (e-mail: sikki@lakeheadu.ca).

E. Alsusa is with the School of Electrical and Electronic Engineering, University of Manchester, Manchester M13 9PL, U.K. (e-mail: e.alsusa@manchester.ac.uk). in the upcoming fifth generation (5G) wireless networks. In overlay CRN, secondary users (SUs) sense the spectrum to identify unoccupied frequency bands, and hence utilize the idle bands to transmit their data [4]. Thus, spectrum sensing is vital to identify the idle bands with high level of accuracy to minimize interference with the licensed users, denoted as primary users (PUs). Towards this goal, several algorithms were developed with the aim of increasing the reliability of the spectrum sensing process [5]-[8]. However in CRNs, multiple SUs might be simultaneously sensing the spectrum and may cooperate to produce more reliable decisions as compared to decisions generated by each SU individually [9]-[12].

The problem of fusing several local decisions into one global decision has received considerable attention in the literature. For example, the authors in [13] considered the fusion problem where the distributed decisions are transmitted over fading channels and derived the optimal and several suboptimal fusion rules, namely, the Chair-Varshney, equal gain combining (EGC), and maximum ratio combining (MRC). The suboptimal rules provide a less computationally demanding solution, but degrades the global decision reliability. Therefore, the authors in [14] purposed an efficient suboptimal fusion rule, denoted as the Max-log, that provides more reliable fusion with less complexity than the optimal. A comprehensive comparison between decode-and-fuse and decode-then-fuse fusion rules is reported in [15] entailing the numerical stability, required variables for fusion, complexity and implementation feasibility. The impact of imperfect channel side information (CSI) on the fusion process is studied in [16]. The performance of fusion systems which apply cooperative orthogonal frequency division multiplexing (OFDM) is studied in [17]. The cooperative differential space time coding is proposed in [18] for sensor networks to mitigate the effect of fading channels. In [19] and [20], the effect of applying decodeand-forward relays is considered and the corresponding fusion rules are derived.

In CRNs, the system performance is highly dependent on the reliability of the individual spectrum detectors. As such, numerous types of spectrum sensing algorithms have been considered such as energy detectors (EDs), compressive sensing, matched filters, radio identification detectors, and spectral correlators [7], [8], [11]. Such algorithms provide a wide range of flexibility for the designer to trade-off reliability and complexity. Nevertheless, the cooperation between multiple spectrum sensors can significantly enhance the reliability of 
CRNs even if low complexity sensors are used. However, the design of optimal fusion rules leads to system instability and introduces additional complexity [14], [15], [19], [20]. Consequently, developing efficient fusion rules is crucial to improve performance without incurring additional computational complexity. Among several potential solutions, the decode-thenfuse rule, also known as the two-stage decision fusion (TSDF) rule, is considered one of the most efficient decision fusion rules because of its low computational complexity and high stability [9]-[13], [21]. ${ }^{\left[R_{2,4}\right]}$ In the first stage of the TSDF rule, the received signals from all SUs are detected and converted to binary decisions, denoted as hard decisions. The second stage is to fuse the hard decisions into a single global decision. Although the TSDF is suboptimal, it can provide reliable error fusion performance that approaches the optimal fusion at high signal to noise ratios (SNRs).

It is reported in the literature that the reliability of the TSDF is mostly dominated by the accuracy of the individual detectors and the channel quality between the SUs and the cognitive radio basestation (CRBS) [19], [20], [22]-[25]. Several modifications have been consequently proposed to enhance the TSDF rule performance by improving the link quality between the SUs and CRBS, such as relays [19], [20], multiple input multiple output (MIMO) [22], [23], and error control coding [24], [25]. However, little attention is given to the detection process in the first stage of the TSDF rule. In particular, it is typically assumed that the spectrum occupancy is balanced, i.e., the probability that the spectrum is occupied or idle is equal. In practice, the spectrum occupancy is actually a highly dynamic parameter [26]-[28], and hence should be taken into consideration.

\section{A. Key Contributions}

Generally speaking, the channel occupancy probability in $\mathrm{CRN}$ is a dynamic factor that is mostly determined by the time and location of the PU network [5], where high occupancy rate is expected during peak working hours and urban areas. Therefore, the detection capability of spectrum sensing systems can be improved if the channel occupancy rate is considered. Considering a CRN with the PU activity model with death rate $\mu_{d}$ and birth date $\mu_{b}$, the channel availability and occupancy rates can be estimated as [5], [26]-[28],

$$
\begin{aligned}
\operatorname{Pr}(\text { idle }) & =\frac{\mu_{d}}{\mu_{d}+\mu_{b}} \\
\operatorname{Pr}(\text { occupied }) & =\frac{\mu_{b}}{\mu_{d}+\mu_{b}}
\end{aligned}
$$

${ }^{\left[R_{2,2}\right]}$ where $\operatorname{Pr}$ (occupied) is the probability that the channel is used by the $\mathrm{PU}$, and $\operatorname{Pr}($ idle $)$ is the palpability that the channel is not used by the PU.

In contrast to existing work, this paper proposes an adaptive TSDF technique for cooperative multihop CRNs. The proposed technique is based on considering the spectrum occupancy statistics into the data detection processes at the relays and the CRBS. The proposed system performance is

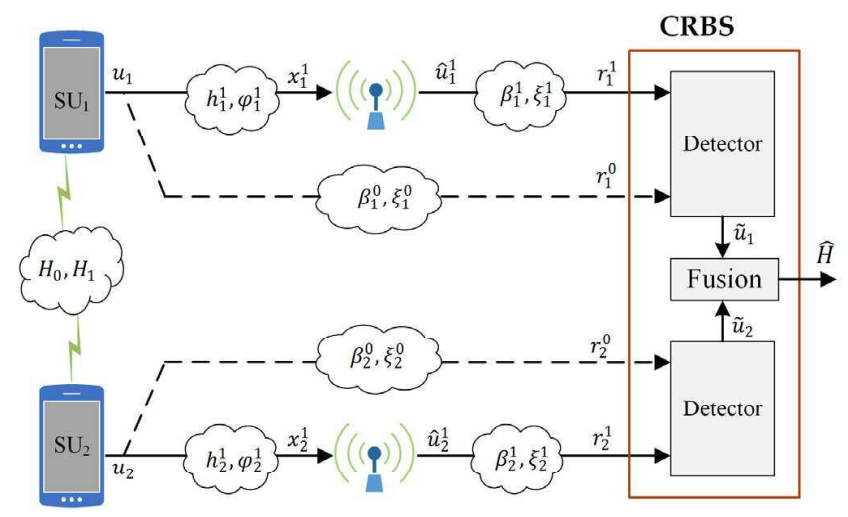

Fig. 1. The considered cooperative cognitive network for $K=2$ and $N=1$.

evaluated in terms of the probability of spectrum sensing errors (PSSE), where analytical and simulation results are presented to verify and demonstrate the system performance under various operating scenarios.

The rest of the paper is organized as follows. Section II presents the system model. Section III discusses the conventional two-stage fusion process for both network structures, i.e., with and without relays. The two-stage rule with dynamic detection threshold is discussed in Section IV, and Section V presents the performance analysis of the fusion process. Sections VI and VII provide the numerical results and conclusion, respectively.

\section{SySTEM MODEL}

In this section, the sensing and transmission models are illustrated separately. For the transmission model, two communication configurations are considered, namely, the single-hop transmission model in which the local decisions of the SUs are sent directly to the CRBS, and the dual-hop transmission model that incorporates DF relays.

\section{A. Spectrum Sensing Model}

The system considered in this work consists of $K$ distributed SUs that simultaneously collect observations about the spectrum of a certain channel. Each SU makes a binary decision about the occupancy of the channel independently. For the $k$ th $\mathrm{SU}$, the received signal during the $l$ th sensing period can be defined as

$$
\left.\begin{array}{cc}
H_{1}: & \chi_{k}[l]=\hbar_{k} x[l]+\bar{\varphi}_{k}[l] \\
H_{0}: & \chi_{k}[l]=\bar{\varphi}_{k}[l]
\end{array}\right\}, k \in\{1,2, \ldots, K\}
$$

where $H_{0}$ and $H_{1}$ denote that the channel is idle and busy, respectively, $x$ is the signal of the $\mathrm{PU}, \hbar_{k}$ is a random variable that captures the effects of the channel fading, and the additive white Gaussian noise (AWGN) $\bar{\varphi}_{k} \sim \mathcal{N}\left(0, \sigma_{\bar{\varphi}}^{2}\right)$. It is worth noting that $\chi_{k} \forall k$ are independent because the AWGN samples and the channel fading coefficients are independent. The received signal at the $k$ th SU $\chi_{k}[l]$ is then used to make a 
hard decision $u_{k} \in\{-1,1\}$. The hard quantization at the $\mathrm{SU}$ is typically considered in power and bandwidth limited systems [11]. The decision-making process of the SU is typically characterized by its detection probability $P_{k}^{\mathrm{d}}$ and false alarm probability $P_{k}^{\mathrm{fa}},[10]-[12]$.

\section{B. Transmission Model}

${ }^{\left[R_{2,3}\right]}$ In the single-hop transmission model, the SUs send their $K$ decisions directly to the CRBS to perform the decision fusion process. In the dual-hop transmission model, $N$ DF relays are used to assist each SU to forward its local decisions to the CRBS. The $K$ decisions transmitted by the SUs may also be received by the CRBS, and hence, $N+1$ signals are received at the CRBS for each SU. The CRBS utilizes the $K \times(N+1)$ signals to make the global decision about the channel status [19], [20]. During the broadcasting phase, an SU broadcasts its decision $u_{k}$, which is received by the CRBS and the cooperating relays. During the relaying phase, each of the relevant relays retransmits a decoded version of $u_{k}$, denoted as $\hat{u}_{k}^{n}$, to the CRBS. The same process is repeated for all SUs and all relays. For all the considered scenarios, the transmission process is assumed to be performed over orthogonal channels [29], [30].

For the dual-hop transmission model, the baseband representation of the received signal at the $n$th relay can be written as

$$
x_{k}^{n}=\sqrt{\mathcal{P}_{k}^{0}} h_{k}^{n} u_{k}+\varphi_{k}^{n}, \quad n \in\{1,2, \ldots, N\}
$$

where $h_{k}^{n} \sim \mathcal{C N}\left(0, \sigma_{h}^{2}\right)$ is the channel fading coefficient, the AWGN $\varphi_{k}^{n} \sim \mathcal{C N}\left(0, \sigma_{\varphi_{k}}^{2}\right)$, and $\mathcal{P}_{k}^{0}$ is the average transmission power. Given that $h_{k}^{n}$ is available at the relay, it can be used to generate the decision variables $z_{k}^{n}$, which are then fed to the detector. Because $u_{k} \in\{-1,1\}$, the detector can be simply expressed as [19], [20]

$$
z_{k}^{n} \triangleq \frac{4 \sqrt{\mathcal{P}_{k}^{0}}}{\sigma_{\varphi_{k}}^{2}} \operatorname{Re}\left[\left(h_{k}^{n}\right)^{*} x_{k}^{n}\right] \underset{\hat{u}_{k}^{n}=-1}{\stackrel{\hat{u}_{k}^{n}=1}{\gtrless} \mathcal{T}_{R, k}^{n}}
$$

where $(\cdot)^{*}$ denotes the complex conjugate and $\mathcal{T}_{R, k}^{n}$ is the detection threshold, which can be used to satisfy a certain criterion.

At the CRBS, the vector $\boldsymbol{r}_{k}=\left[r_{k}^{0}, r_{k}^{1}, r_{k}^{2}, \ldots, r_{k}^{N}\right]$ is received from the $k$ th $\mathrm{SU}$, where $r_{k}^{0}$ represents the direct signal while $r_{k}^{n}, n \in\{1, \ldots, N\}$, represents the $n$th relayed signal,

$$
r_{k}^{n}=\sqrt{\mathcal{P}_{k}^{n}} \hat{u}_{k}^{n} \beta_{k}^{n}+\xi_{k}^{n}, n \in\{0,1, \ldots, N\}
$$

where $\beta_{k}^{n} \sim \mathcal{C N}\left(0, \sigma_{\beta_{k}}^{2}\right)$ is the fading coefficient, $\xi_{k}^{n} \sim$ $\mathcal{C N}\left(0, \sigma_{\xi_{k}}^{2}\right)$ is the AWGN, and $\mathcal{P}_{k}^{n}$ is the average transmission power. It is worth noting that in (5), $\hat{u}_{k}^{0}=u_{k}$.

${ }^{\left[R_{2,3}\right]}$ For the single-hop transmission model, the received signal of the $k$ th SU at the CRBS is reduced to

$$
r_{k}=\sqrt{\mathcal{P}_{k}} u_{k} \beta_{k}+\xi_{k}
$$

where the relay index is simply dropped. The considered system model is depicted in Fig. 1 for the case of $K=2$ and $N=1$.

\section{Two-Stage Decision Fusion USing DF RElays}

${ }^{\left[R_{2,3}\right]}$ In the conventional two-stage decision fusion (TSDF) rule with single-hop transmission model, the received signals that correspond to the local decisions are first detected at the CRBS to create an estimated version of the local decisions, denoted as $\tilde{\mathbf{u}}=\left[\tilde{u}_{1}, \tilde{u}_{2}, \ldots, \tilde{u}_{K}\right], \tilde{u}_{k} \in\{-1,1\}$. In the second stage, all the decisions $\tilde{u}_{1}, \tilde{u}_{2}, \ldots, \tilde{u}_{K}$ are fused to generate the global decision. In the presence of dual-hop transmission, the received signals that correspond to a particular sensor and the associated relays are combined and detected to generate a single binary value, then, all binary values that correspond to the $K$ sensors are fused in the second stage to generate the global decision. Therefore, the TSDF rule requires a detector at each relay for the DF process, and another detector is required at the CRBS prior the fusion process. The detector at the CRBS is similar to (4) except that the detection threshold is denoted as $\mathcal{T}_{C, k}$.

In decision fusion problems, the probability of fusion error (PFE) is widely used as the metric to evaluate the performance of various fusion rules [19], [20], [31]. In spectrum sensing applications, the PFE is equivalent to the PSSE, which can be expressed as

$$
P_{\mathrm{e}}=P_{F A} \operatorname{Pr}\left(H_{0}\right)+\left(1-P_{D}\right) \operatorname{Pr}\left(H_{1}\right)
$$

where $P_{D}$ and $P_{F A}$ are the global probabilities of detection and false alarm, respectively. The detection thresholds at the fusion center $\mathcal{T}_{C}=\left[\mathcal{T}_{C, 1}, \mathcal{T}_{C, 2}, \ldots, \mathcal{T}_{C, K}\right]$ and the relays associated with the $k$ th SU $\mathcal{T}_{R, k}=\left[\mathcal{T}_{R, k}^{1}, \mathcal{T}_{R, k}^{2}, \ldots, \mathcal{T}_{R, k}^{N}\right]$ can be selected to minimize PSSE,

$$
\left[\mathcal{T}_{R, 1} \ldots \mathcal{T}_{R, K}, \mathcal{T}_{C}\right]=\arg \min _{\left\{\mathcal{T}_{R, 1} \ldots \mathcal{T}_{R, K}, \mathcal{T}_{C}\right\}} P_{\mathrm{e}}
$$

As it can be noted from (8), minimizing $P_{\mathrm{e}}$ is a multidimensional optimization problem with intractable solution [31]. Hence, most of the work reported in the literature that is based on the TSDF rule considers that $\mathcal{T}_{C, k}=\mathcal{T}_{R, k}^{n} \triangleq \tau_{0}=0$ $\forall\{k, n\}$ [9]-[13], [21], which significantly reduces the complexity, but degrades PSSE. Therefore, the detectors at the relays and CRBS described by (4) become the conventional distributed maximum likelihood (ML) detectors. In such scenarios, the detectors will minimize the probability of error of the corresponding transmission segment in the network given that the transmitted binary signals over that link have equal prior probabilities. For example, the detector at the relay that corresponds to the $k$ th SU will minimize $\operatorname{Pr}\left(\tilde{u}_{k} \neq u_{k}\right)$ given that $\operatorname{Pr}\left(u_{k}=1\right)=0.5$. However, the spectrum occupancy probability is highly dynamic, i.e., $\operatorname{Pr}\left(H_{1}\right) \neq \operatorname{Pr}\left(H_{0}\right)$, and hence $\operatorname{Pr}\left(u_{k}=1\right) \neq \operatorname{Pr}\left(u_{k}=-1\right)$. Consequently, the detectors in the system will not be optimal in terms of minimizing PSSE, nor the transmission error probability $\operatorname{Pr}\left(\tilde{u}_{k} \neq u_{k}\right)$. In this sequel, it is worth evaluating the performance of the system when the detectors are designed to minimize the transmission error probability in a distributed manner. 
Therefore, PSSE in this work is evaluated given that $\mathcal{T}_{R, k}$ is chosen to minimize the error probability of the SU-relay link, while $\mathcal{T}_{C, k}$ is chosen to minimize the probability of error of the SU-CRBS link. Therefore, the maximum a posterior probability (MAP) detector can be used at the relay which is given in (4) where the threshold $\mathcal{T}_{R, k}$ is given by

$$
\mathcal{T}_{R, k}=\ln \left(\frac{P_{0, k}}{P_{1, k}}\right)
$$

where $P_{0, k}=\operatorname{Pr}\left(u_{k}=-1\right)$ and $P_{1, k}=\operatorname{Pr}\left(u_{k}=1\right)$. Thus, for the special case when $P_{0, k}=P_{1, k}$, the threshold $\mathcal{T}_{R, k}=0$. The a priori probabilities of $u_{k}$ are defined as

$$
\begin{gathered}
P_{1, k}=P_{k}^{\mathrm{fa}} \operatorname{Pr}\left(H_{0}\right)+P_{k}^{\mathrm{d}} \operatorname{Pr}\left(H_{1}\right) \\
P_{0, k}=1-P_{1, k} .
\end{gathered}
$$

For the general case where $\mathcal{T}_{R, k} \neq 0$, the SU-relay link can be considered as a binary asymmetric channel with transition probabilities $\rho_{k}^{n} \triangleq \operatorname{Pr}\left(\hat{u}_{k}^{n}=1 \mid u_{k}=-1\right)$ and $\varepsilon_{k}^{n} \triangleq$ $\operatorname{Pr}\left(\hat{u}_{k}^{n}=-1 \mid u_{k}=1\right)$, which are derived in Appendix A.

At the CRBS, the detection stage is different from the one at the relay because $N+1$ signals are received for each SU. Because the direct and relayed signals can be considered mutually independent, the MAP detector that minimizes the probability of error can be written as

$$
\frac{\prod_{n=0}^{N} f\left(r_{k}^{n} \mid u_{k}=1\right)}{\prod_{n=0}^{N} f\left(r_{k}^{n} \mid u_{k}=-1\right)} \stackrel{\tilde{u}_{k}=-1}{\tilde{u}_{k}=1} \frac{P_{0, k}}{P_{1, k}}
$$

where $f\left(r_{k}^{n} \mid u_{k}\right)$ is the conditional PDF of $r_{k}^{n}$ at the CRBS. After some straightforward manipulations, the MAP detector can be written as

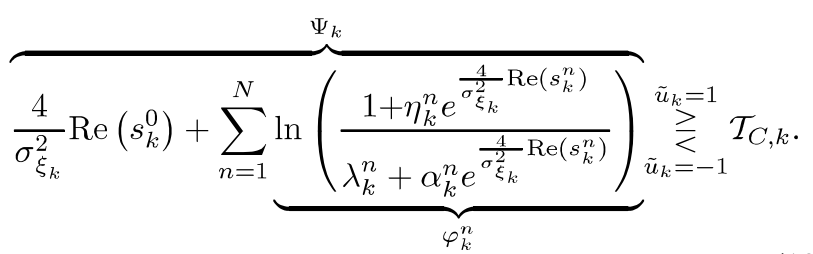

The variables in (13) are defined as, $s_{k}^{n}=r_{k}^{n}\left(\beta_{k}^{n}\right)^{*}, \eta_{k}^{n}=$ $\frac{1-\rho_{k}^{n}}{\rho_{k}^{n}}, \alpha_{k}^{n}=\frac{\varepsilon_{k}^{n}}{\rho_{k}^{n}}, \lambda_{k}^{n}=\frac{1-\varepsilon_{k}^{n}}{\rho_{k}^{n}}$, and $\mathcal{T}_{C, k}=\ln \frac{P_{0, k}}{P_{1}, k}$. As can be noted from (13), the summation argument $\varphi_{k}^{n}$ is nonlinear and might encounter computational instability at high SNRs. To simplify the detection process, piecewise linear approximation can be used [19]

$$
\varphi_{k}^{n} \approx\left\{\begin{array}{cc}
d_{k}, & y_{k}^{n}<c_{1} \\
g_{k} y_{k}+l_{k}, & c_{1}<y_{k}^{n}<c_{2} \\
b_{k}, & y_{k}^{n}>c_{2}
\end{array} .\right.
$$

In (14), $y_{k}^{n}=\left(4 / \sigma_{\xi_{k}}^{2}\right) \operatorname{Re}\left(s_{k}^{n}\right), b_{k}=\ln \left(\frac{\eta_{k}^{n}}{\alpha_{k}^{n}}\right), d_{k}=$ $\ln \left(\frac{1}{\lambda_{k}^{n}}\right), g_{k}=\frac{\left(\lambda_{k}^{n} e^{c_{0}}-1\right)\left(\eta_{k}^{n}-\alpha_{k}^{n} e^{c_{0}}\right)}{e^{c_{0}}\left(\eta_{k}^{n} \lambda_{k}^{n}-\alpha_{k}^{n}\right)}, l_{k}=0.5 \ln \left(\frac{\eta_{k}^{n}}{\alpha_{k}^{n} \lambda_{k}^{n}}\right)-$ $g_{k} \ln \left(\frac{\lambda_{k}^{n} e^{c_{0}}-1}{\eta_{k}^{n}-\alpha_{k}^{n} e^{c_{0}}}\right), c_{0}=\ln \left(\sqrt{\frac{\eta_{k}^{n}}{\alpha_{k}^{n} \lambda_{k}^{n}}}\right), c_{1}=\frac{d_{k}-l_{k}}{g_{k}}$, and $c_{2}=\frac{b_{k}-l_{k}}{g_{k}}$. It is worth noting that for the case of ideal relays, $\left\{\rho_{k}^{n}, \varepsilon_{k}^{n}\right\}=0$, the decision metric in (13) is reduced to the conventional MRC,

$$
\begin{aligned}
\Psi_{k} & =\frac{4}{\sigma_{\xi_{k}}^{2}} \operatorname{Re}\left(s_{k}^{0}\right)+\sum_{n=1}^{N}\left(\frac{\rho_{k}^{n}+\left(1-\rho_{k}^{n}\right) e^{\frac{4}{\sigma_{\xi_{k}}^{2}} \operatorname{Re}\left(s_{k}^{n}\right)}}{\left(1-\varepsilon_{k}^{n}\right)+\varepsilon_{k}^{n} e^{\frac{4}{\sigma_{\xi_{k}}^{2}} \operatorname{Re}\left(s_{k}^{n}\right)}}\right) \\
& =\frac{4}{\sigma_{\xi_{k}}^{2}} \sum_{n=0}^{N} \operatorname{Re}\left(r_{k}^{n}\left(\beta_{k}^{n}\right)^{*}\right) .
\end{aligned}
$$

Once the detection stage is completed, the $K$ resultant decisions are sent to the second stage for fusion to produce the global decision. In the literature, several fusion rules are proposed for fusing binary local decisions, examples for such rules are the $\bar{K}$ out of $K$, AND, OR, majority voting algorithm, and Chair-Varshney [11]. Due to its efficiency, we consider the Chair-Varshney rule [9], [10], [13], [14], [21] to generate the global decision, which can be expressed as

$$
\sum_{k \mid \tilde{u}_{k}=1} \ln \left(\frac{P_{k}^{\mathrm{d}}}{P_{k}^{\mathrm{fa}}}\right)+\sum_{k \mid \tilde{u}_{k}=-1} \ln \left(\frac{1-P_{k}^{\mathrm{d}}}{1-P_{k}^{\mathrm{fa}}}\right) \underset{H_{1}}{\stackrel{H_{0}}{>}} \tau_{G} .
$$

The fusion threshold $\tau_{G}$ can be used to control $P_{D}$ and $P_{F A}$.

${ }^{\left[R_{2,1}\right]}$ As can be noted from the decision variable given in (15), the knowledge of the instantaneous channel coefficients is necessary to perform the optimum detection for the received symbols. Such requirement is typical and does not pose any major challenge for the system realization. However, the situation is different for the fusion process described (16) that requires the knowledge statistical behavior of the spectrum sensors, which is represented by $P_{k}^{\mathrm{d}}$ and $P_{k}^{\mathrm{fa}} \forall k$.

\section{Probability of Spectrum Sensing Error (PSSE) ANALYSIS}

Given that all SUs use the same spectrum detection algorithm, it is acceptable to assume that $P_{1}^{\mathrm{d} / \mathrm{fa}} \approx P_{2}^{\mathrm{d} / \mathrm{fa}} \approx \cdots \approx$ $P_{K}^{\mathrm{d} / \mathrm{fa}}[9]-[13],[21]$, and hence $P_{k}^{\mathrm{d} / \mathrm{fa}}$ can be written as $P^{\mathrm{d} / \mathrm{fa}}$ after dropping the $\mathrm{SU}$ index. Therefore, the fusion rule given in (16) can be simplified to

$$
K_{1} \ln \left(\frac{P^{\mathrm{d}}}{P^{\mathrm{fa}}}\right)+\left(K-K_{1}\right) \ln \left(\frac{1-P^{\mathrm{d}}}{1-P^{\mathrm{fa}}}\right) \underset{H_{1}}{\stackrel{H_{0}}{\lessgtr}} \tau_{G}
$$

where $K_{1}=\frac{1}{2} \sum_{k}\left(\tilde{u}_{k}+1\right)$, i.e., the total number of decisions that has $\tilde{u}_{k} \stackrel{1}{=}$. After some straightforward manipulations (17) can be written as

$$
K_{1} \underset{H_{1}}{\stackrel{H_{0}}{\lessgtr}} \frac{\tau_{G}-K \ln \left(\frac{1-P^{\mathrm{d}}}{1-P^{\mathrm{fa}}}\right)}{\ln \left(\frac{P^{\mathrm{d}}\left(1-P^{\mathrm{fa}}\right)}{P^{\mathrm{fa}}\left(1-P^{\mathrm{d}}\right)}\right)} \triangleq \chi_{g} .
$$

As depicted in (18), the fusion process is based on comparing the total number of local decisions with value 1 , to the threshold $\chi_{g}$. Apparently, $K_{1} \in\{0,1, \ldots, K\}$ is a random integer produced as the sum of $K$ independent Bernoulli trials each of which has probability of success $p_{k \mid H_{1 / 0}}=\operatorname{Pr}\left(\tilde{u}_{k}=1 \mid H_{1 / 0}\right)$. Thus, $K_{1}$ is a conditionally Poisson-binomial random variable, 
$K_{1} \mid H_{1} \sim P B\left(K, p_{k \mid H_{1}}\right)$ and $K_{1} \mid H_{0} \sim P B\left(K, p_{k \mid H_{0}}\right)$, where

$$
\begin{aligned}
p_{k \mid H_{1 / 0}}= & \operatorname{Pr}\left(\tilde{u}_{k}=1 \mid H_{1 / 0}\right) \\
= & \operatorname{Pr}\left(\tilde{u}_{k}=1 \mid u_{k}=1\right) P^{\mathrm{d} / \mathrm{fa}} \\
& +\operatorname{Pr}\left(\tilde{u}_{k}=1 \mid u_{k}=-1\right)\left(1-P^{\mathrm{d} / \mathrm{fa}}\right) .
\end{aligned}
$$

The pairwise error probability $\operatorname{Pr}\left(\tilde{u}_{k} \mid u_{k}\right)$ is derived in Appendix B. Moreover, because $K_{1}$ can take only integer values, an integer threshold $K_{T}=\left\lceil\chi_{g}\right\rceil$ can be used where $\lceil\cdot\rceil$ is the ceiling function.

Using the Poisson-binomial cumulative distribution function (CDF) of $K_{1}$, the global $P_{D}$ and $P_{F A}$ can be computed as [32]

$$
\begin{aligned}
P_{D / F A}= & \operatorname{Pr}\left(K_{1} \geq K_{T} \mid H_{1 / 0}\right) \\
= & 1-\frac{K_{T}}{K+1}-\frac{1}{K+1} \sum_{i=1}^{K}\left(\frac{1-e^{-j \frac{2 \pi i K_{T}}{K+1}}}{1-e^{-j \frac{2 \pi i}{K+1}}}\right. \\
& \left.\quad \times \prod_{k=1}^{K}\left[1+p_{k \mid H_{1 / 0}}\left(e^{j \frac{2 \pi i}{K+1}}-1\right)\right]\right) .(20)
\end{aligned}
$$

It is worth noting that for the case where all fading channels have the same statistical characteristics, the variable $p_{k \mid H_{1 / 0}}$ becomes equal for all detected signals, and thus, the $\mathrm{SU}$ index can be dropped, i.e., $p_{k \mid H_{1 / 0}} \triangleq p_{1 / 0} \forall k$. Consequently, $K_{1}$ becomes conditionally binomial random variable, and $P_{D / F A}$ can be expressed as

$$
P_{D / F A}=\sum_{k=K_{1}}^{K}\left(\begin{array}{c}
K \\
k
\end{array}\right)\left(p_{1 / 0}\right)^{k}\left(1-p_{1 / 0}\right)^{K-k} .
$$

\section{A. The proposed adaptive detection process}

The main advantage of the derived analysis is that it enables the evaluation of the PSSE for any set of arbitrary detection thresholds $\mathcal{T}_{R, 1} \ldots \mathcal{T}_{R, K}, \mathcal{T}_{C}$. However, because solving the optimization problem in (8) is intractable, a suboptimal solution will be considered based on the reliability of the decisions made at the SUs as well as the statistics of the spectrum occupancy. By noting that improving the SU-CRBS link does not necessarily improve the PSSE, using a suboptimal detection threshold at the relays and CRBS may offer better PSSE. Therefore, the detection thresholds should be selected adaptively such that

$$
\left[\mathcal{T}_{R, 1} \ldots \mathcal{T}_{R, K}, \mathcal{T}_{C}\right]=\arg \min _{\left\{\tau_{0}, \mathcal{T}_{C, k}, \mathcal{T}_{R, k}\right\}} P_{\mathrm{e}}, \forall k
$$

In the case that all channels are independent and identically distributed (iid), and the sensors have equivalent characteristics, all detection thresholds in the network will be identical, and hence $\mathcal{T}_{C, k}=\mathcal{T}_{R, k}^{n} \triangleq \tau_{A}$ and the adaptive threshold in (22) can be simplified to

$$
\left[\tau_{V}\right]=\arg \min _{\left\{\tau_{0}, \tau_{A}\right\}} P_{\mathrm{e}} .
$$

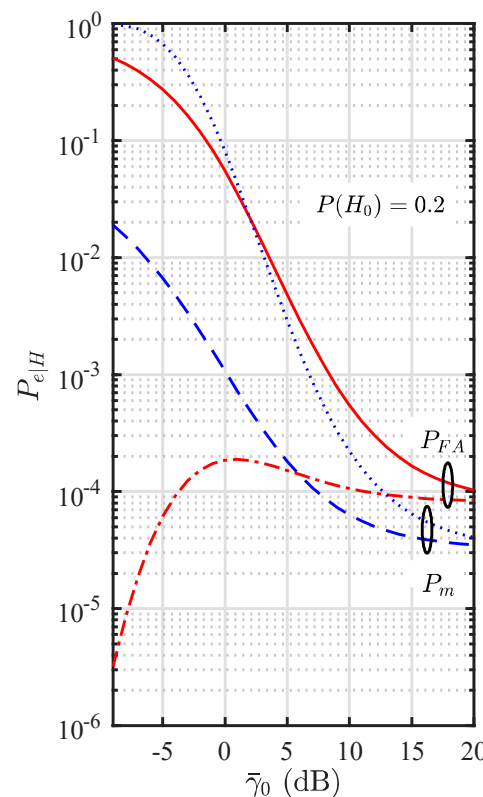

a)

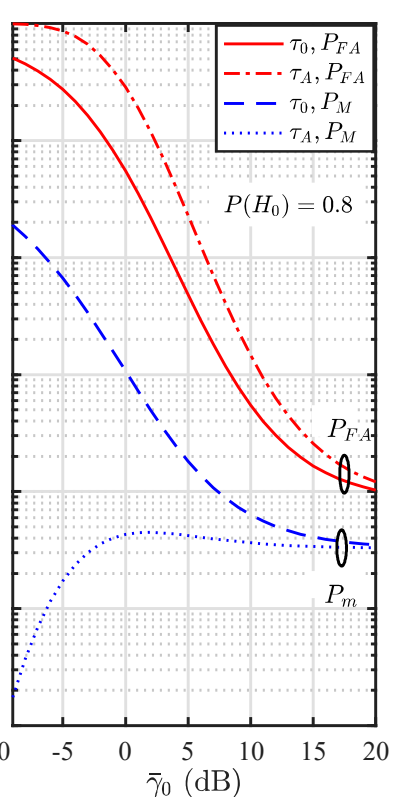

b)
Fig. 3. The conditional PFE for $\tau_{0}=0$ and dynamic $\tau_{A}$ for different values of $P_{k}^{\mathrm{d}}=0.8, P_{k}^{\mathrm{fa}}=0.05, K=8, N=0$.

\section{NuMERICAL RESUlTS}

The performance of the proposed cognitive radio system is evaluated in terms of PSSE given that $K$ SUs are sensing a certain spectrum band to determine its availability for possible transmission. Each of the $K$ users is associated with a relay that decodes and forwards the decisions to the CRBS, where the fusion process is performed. The simulation results are obtained using Monte Carlo simulation where each simulation run consists of $10^{6}$ realization. All channels are considered iid flat Rayleigh fading channels, thus $\left\{\beta_{k}^{n}, h_{k}^{n}\right\} \sim$ $\mathcal{C N}(0,1) \forall\{k, n\}$. The global fusion threshold $\tau_{G}=0$ for all the considered scenarios. The results are presented for both thresholds $\tau_{A}$ and $\tau_{0}$. For fair comparison, the total average transmission power for a $\mathrm{SU}$ and the associated relays is fixed, i.e., $\mathcal{P}_{k}=\sum_{n=0}^{N} \mathcal{P}_{k}^{n}$. Moreover, the average SNR for SU-torelay link is $\bar{\gamma}_{S-R} \triangleq \bar{\gamma}_{0}$, and the average SNR for SU-toCRBS link is $\bar{\gamma}_{S-B S}=0.5 \bar{\gamma}_{0}$ to include the effect of distance.

Fig. 2 presents the PSSE of the considered system without relays, $N=0$. As it can be noted from the figure, using $\tau_{A}$ managed to improve PSSE for $\operatorname{Pr}\left(H_{1}\right)=0.2$ and 0.5 , while for $\operatorname{Pr}\left(H_{1}\right)=0.8$ the PSSE experienced some degradation. Moreover, the improvement/degradation is proportional to the number of SUs $K$. The PSSE degradation using $\tau_{A}$ observed for $\operatorname{Pr}\left(H_{1}\right)=0.8$ is due to the fact that the decisions made by the SUs are generally not reliable when the sensed spectrum is occupied, i.e., $P_{k}^{\mathrm{m}} \triangleq \operatorname{Pr}\left(u_{k}=0 \mid H_{1}\right)>\operatorname{Pr}\left(u_{k}=1 \mid H_{0}\right)$. Thus, the global probability of miss $P_{M}$ is enhanced at the expenses of $P_{F A}$; however, the values of $P_{F A}$ increases substantially and hence becomes the dominant factor which 


\section{drives the PSSE to increase.}

Fig. 3 shows an example for the conditional PSSE, $P_{e \mid H_{0}}=$ $P_{F A}$ and $P_{e \mid H_{1}}=P_{M}$, using $K=8$. It is also worth noting that at high SNRs, the PSSE using $\tau_{A}$ and $\tau_{0}$ converges to an error floor that is determined by $K$ and $\operatorname{Pr}\left(H_{1}\right)$. Moreover, it can be noted that the error floor decreases when $\operatorname{Pr}\left(H_{1}\right)$ or $K$ increases. Finally, the figure shows that the simulation results corroborate the analytic solutions for all the considered cases.

Fig. 4 shows the effect of using $\tau_{A}$ on PSSE when each $\mathrm{SU}$ is associated with a single $\mathrm{DF}$ relay, i.e., $N=1$. The detection process at the relay and CRBS is performed either using $\mathcal{T}_{R, k}=\mathcal{T}_{C, k}=\tau_{A}$ or $\mathcal{T}_{C, k}=\mathcal{T}_{R, k}=\tau_{0}=0$. As it can be noted from the figure, the general trends of PSSE are following the $N=0$ case depicted in Fig. 2. However, unlike the $N=0$ case, the improvement gained using $\tau_{A}$ is generally smaller and some degradation is obtained the case of $\operatorname{Pr}\left(H_{1}\right)=0.2$. The error floor level for both values of $N$ is equivalent because the effect of relays on PSSE is negligible at high SNRs. The figure also shows the correspondence between the analytical and simulation results.

Fig. 5 shows the PSSE using $\tau_{A}$ for different values of $K$ when $N=0,1$. As can be noticed from the figure, applying a relay managed to improve the PSSE at moderate and high ranges of SNR but some degradation occurred at low SNR values. This behavior can be attributed to the fact that the probability of detection error at the DF relay is high at low SNR, and thus errors in the system increase. However, the overall performance of the system is very poor at low SNR in all cases, and thus the CRN would only work in good SNR conditions. The relays do not affect the error floor which depends on the number of sensors $K$ and $\operatorname{Pr}\left(H_{1}\right)$ as aforementioned.

${ }^{\left[R_{2,5}\right]}$ Figs. 6 and 7 show the effect of increasing $P_{k}^{\mathrm{d}}$ on the PSSE for the considered sensing system with and without relays, i.e., $N=0$ and $N=1$. As can be noted from the figures, increasing $P_{k}^{\mathrm{d}}$ effectively improves the PSSE, and the performance gain obtained by applying $\tau_{A}$ decreases for $\operatorname{Pr}\left(H_{1}\right)=0.2$ and 0.5. On the other hand, the performance degradation for the case of $\operatorname{Pr}\left(H_{1}\right)=0.8$ increases as $P_{k}^{\mathrm{d}}$ increases.

To evaluate the gain obtained using the adaptive threshold $\tau_{V}$ as compared to the conventional threshold $\tau_{0}$, Table I shows the relative PSSE, which is defined as the ratio of the PSSE using $\tau_{0}$ over the PSSE using $\tau_{V}$. As it can be noted form the table, the adaptation process may offer a substantial PSSE reduction of 9 fold in certain scenarios. The entries were the relative PSSE is unity corresponds to the cases were $\tau_{V}=\tau_{0}$.

\section{CONCLUSION AND FUTURE WORK}

In this work, the cooperative spectrum sensing system was studied, where SUs sense a certain spectrum band for possible transmission and transmit their decisions to the CRBS to generate the global decision. The performance of the twostage fusion rule was analyzed in terms of PSSE, where the effects of introducing DF relays and applying adaptive threshold are investigated. Simulation results confirmed the derived analytical expressions for the PSSE for different simulation parameters. In addition, it was observed that the PSSE suffers from error floor at high values of SNR, where 


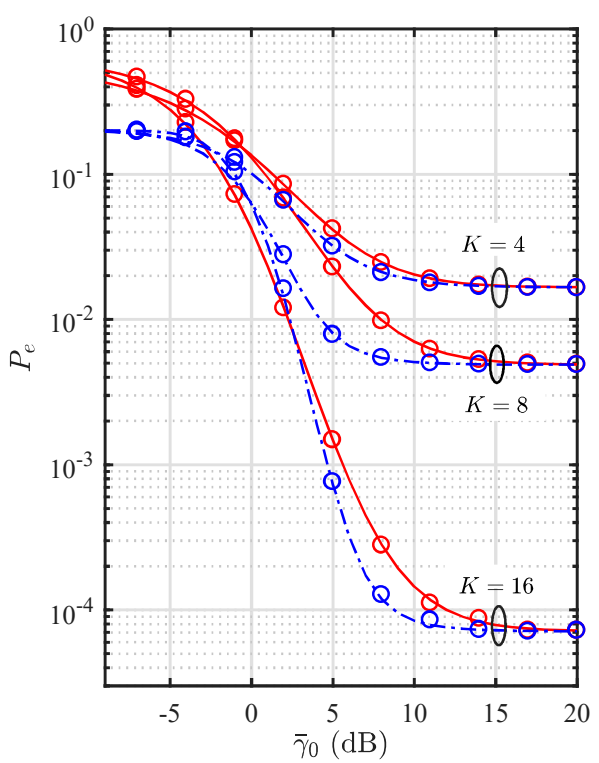

a)

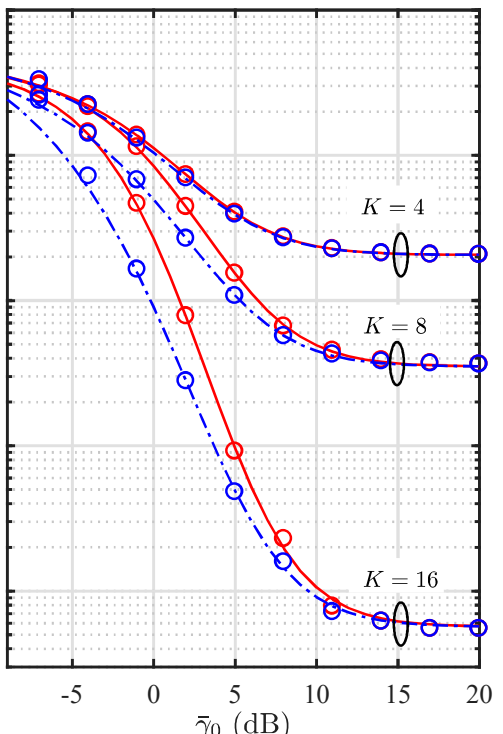

b)

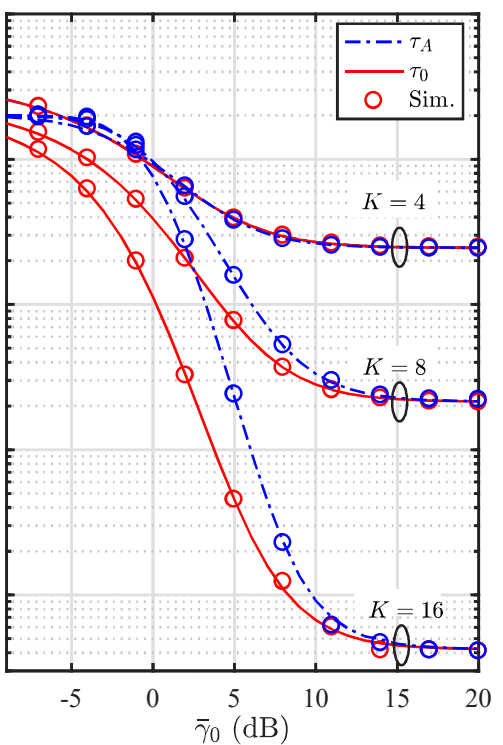

c)

Fig. 4. The PEF for a system with $\tau_{0}=0$ and dynamic $\tau_{A}$ for different values of $K, P_{k}^{\mathrm{d}}=0.8, P_{k}^{\mathrm{fa}}=0.05, N=1:$ a) $\left.\operatorname{Pr}\left(H_{1}\right)=0.2, \mathrm{~b}\right) \operatorname{Pr}\left(H_{1}\right)=0.5$, c) $\operatorname{Pr}\left(H_{1}\right)=0.8$.

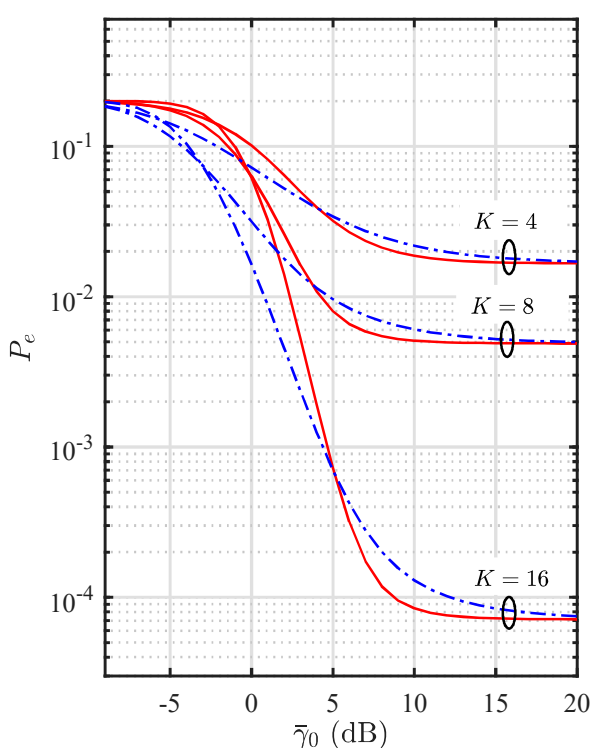

a)

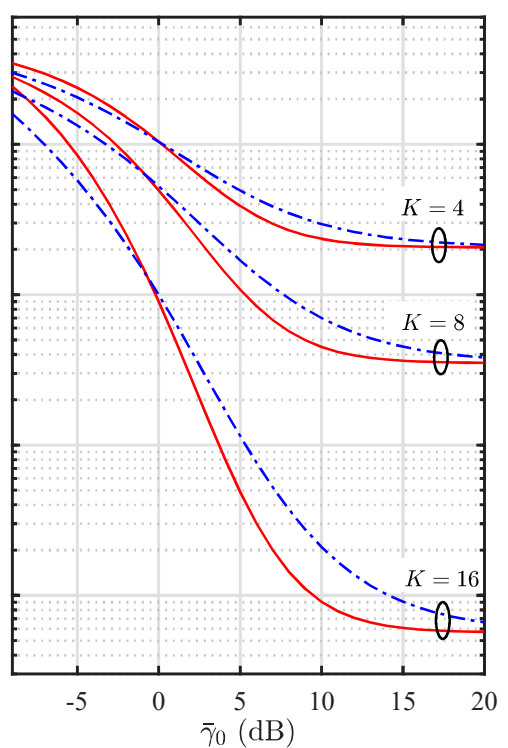

b)

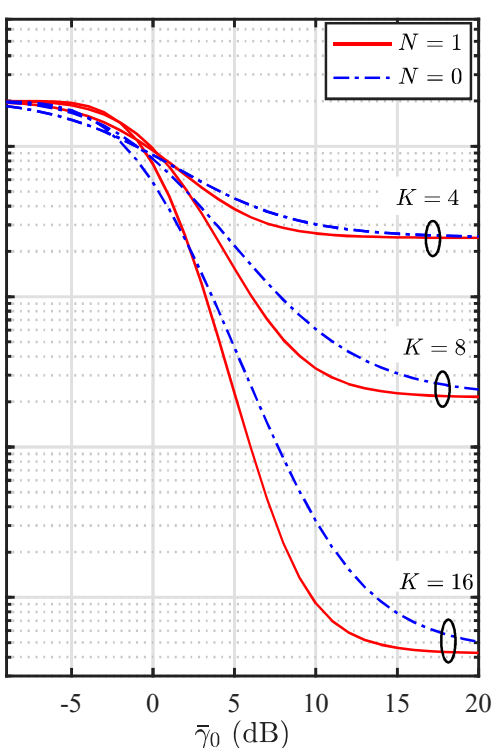

c)

Fig. 5. The PEF for a system applies the dynamic detection threshold $\tau_{A}$ with $N=0$ and $N=1$ for different values of $K, P_{k}^{\mathrm{d}}=0.8, P_{k}^{\mathrm{fa}}=0.05$ : a) $\operatorname{Pr}\left(H_{1}\right)=0.2$, b) $\left.\operatorname{Pr}\left(H_{1}\right)=0.5, \mathrm{c}\right) \operatorname{Pr}\left(H_{1}\right)=0.8$. 


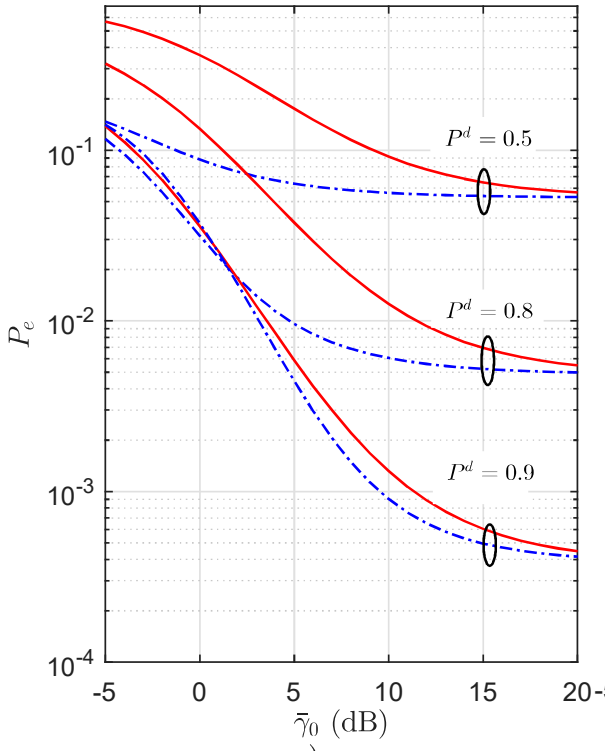

a)

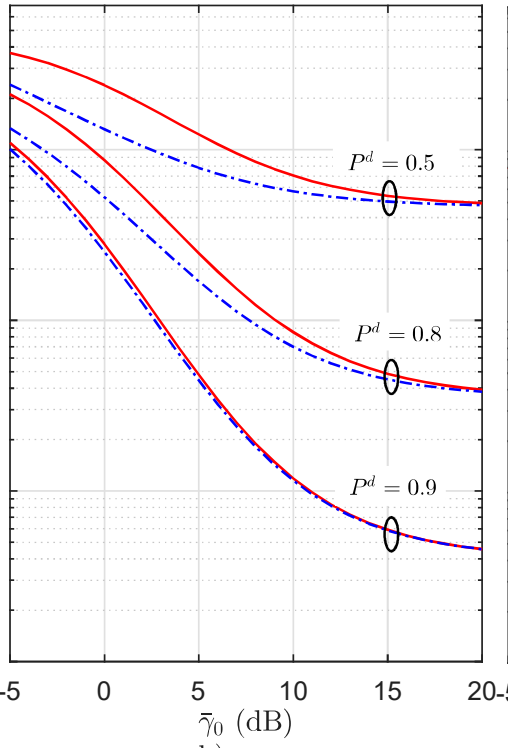

b)

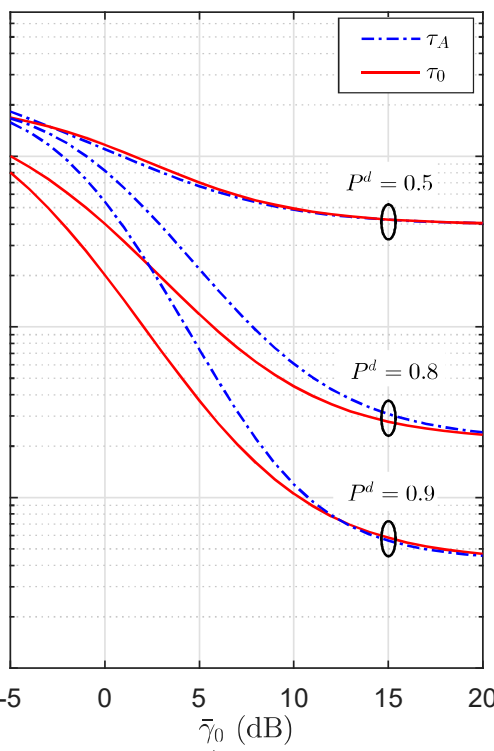

c)

Fig. 6. The PEF for a system with $\tau_{0}=0$ and dynamic $\tau_{A}$ for different values of $P_{k}^{\mathrm{d}}, K=8, N=0, P_{k}^{\mathrm{fa}}=0.05$ : a) $\left.\operatorname{Pr}\left(H_{1}\right)=0.2, \mathrm{~b}\right) \operatorname{Pr}\left(H_{1}\right)=0.5$, c) $\operatorname{Pr}\left(H_{1}\right)=0.8$.

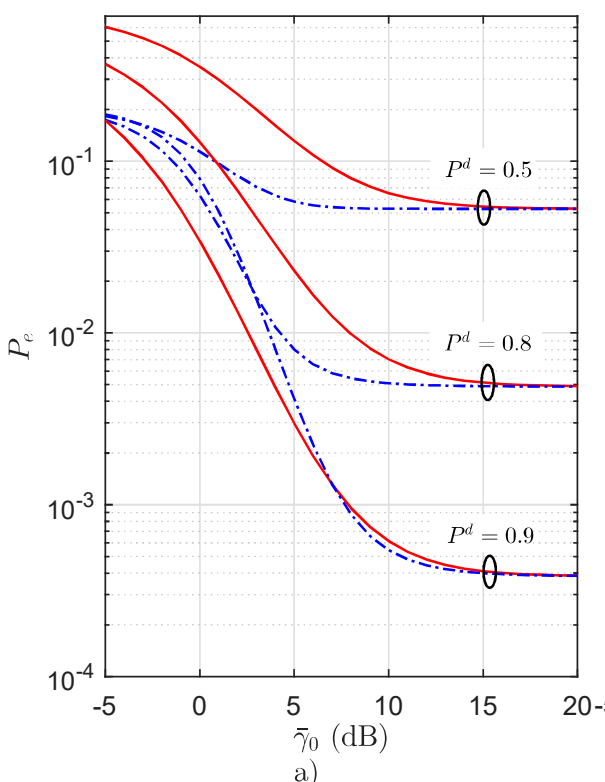

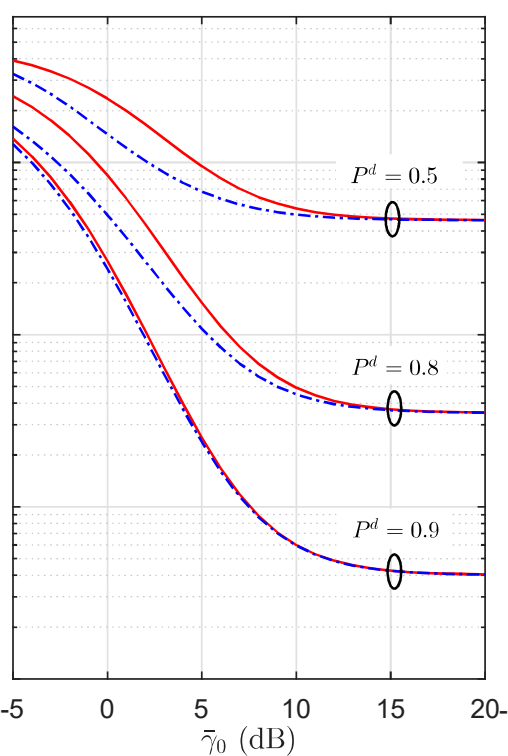

b)

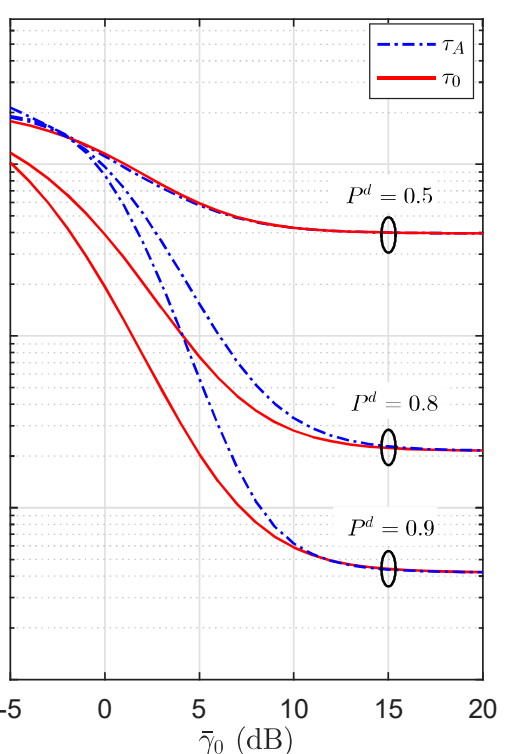

c)

Fig. 7. The PEF for a system with $\tau_{0}=0$ and dynamic $\tau_{A}$ for different values of $P_{k}^{\mathrm{d}}, K=8, N=1, P_{k}^{\mathrm{fa}}=0.05:$ a) $\operatorname{Pr}\left(H_{1}\right)=0.2$, b) $\operatorname{Pr}\left(H_{1}\right)=0.5$, c) $\operatorname{Pr}\left(H_{1}\right)=0.8$ 
the floor is determined by the number of SUs $K$, the sensing capability of the $\mathrm{SU}, P_{k}^{\mathrm{fa}}$ and $P_{k}^{\mathrm{d}}$, and the probability of channel occupancy $\operatorname{Pr}\left(H_{1}\right)$. The results proved that considering the adaptive detection threshold at relays and/or the CRBS improves $P_{M}$ but degrades $P_{F A}$, where this trade-off led to overall improvement for PSSE in some scenarios in which the channel is most probably idle, $\operatorname{Pr}\left(H_{1}\right) \leq 0.5$. On the contrary, some performance degradation was obtained for the case of $\operatorname{Pr}\left(H_{1}\right)>0.5$, in which the degraded $P_{F A}$ dominates the fusion errors.

${ }^{\left[R_{2,1}\right]}$ In future work, the performance of the considered system will be evaluated when the SUs make soft decisions about the spectrum occupancy. In such scenarios, the decisions are analog, and hence, provide more information for the fusion process.

ApPendix A: DeRIVATION OF PEP $\operatorname{Pr}\left(\hat{u}_{k}^{n} \mid u_{k}\right)$ AT THE RELAY

The transition error probabilities at the relay can be written as

$$
\varepsilon_{k}^{n}=\int_{-\infty}^{\mathcal{T}_{R, k}} f\left(z_{k}^{n} \mid u_{k}=1\right) d z_{k}^{n}
$$

and

$$
\rho_{k}^{n}=\int_{\mathcal{T}_{R, k}}^{\infty} f\left(z_{k}^{n} \mid u_{k}=-1\right) d z_{k}^{n} .
$$

The conditional probability density function (PDF) of $z_{k}^{n} \mid u_{k}$ (4) is given by [14], [19], [20]

$$
f\left(z_{k}^{n} \mid u_{k}\right)=\frac{v_{1} v_{2}}{v_{1}+v_{2}}\left[e^{v_{2} z_{k}^{n}} \Phi\left(-z_{k}^{n}\right)+e^{-v_{1} z_{k}^{n}} \Phi\left(z_{k}^{n}\right)\right]
$$

where $\Phi(\cdot)$ is the unit step function and $v_{i}, i \in\{1,2\}$, can be expressed as

$$
v_{i}=\sqrt{w_{k}^{2}+\frac{1}{C_{k}^{2}\left(\sigma_{x}^{2} \sigma_{h}^{2}-\mu_{x, h}^{2}\right)}}+(-1)^{i} w_{k}
$$

and

$$
\begin{aligned}
w_{k} & =\frac{\mu_{x, h}}{C_{k}\left(\sigma_{x}^{2} \sigma_{h}^{2}-\mu_{x, h}^{2}\right)}, C_{k}=\frac{2}{\sigma_{\varphi_{k}}^{2}}, \mu_{x, h}=\sqrt{\mathcal{P}_{k}} \sigma_{h}^{2} \\
\sigma_{x}^{2} & =\mathcal{P}_{k} \sigma_{h}^{2}+\sigma_{\varphi_{k}}^{2} .
\end{aligned}
$$

Evaluating the integrals in (24) and (25) directly gives

$$
\varepsilon_{k}^{n}=\left\{\begin{array}{cc}
\frac{v_{1}}{v_{1}+v_{2}} e^{v_{2} \mathcal{T}_{R, k}}, & \mathcal{T}_{R, k} \leq 0 \\
1-\frac{v_{2}}{v_{1}+v_{2}} e^{-v_{1}} \mathcal{T}_{R, k}, & \mathcal{T}_{R, k}>0
\end{array}\right.
$$

and

$$
\rho_{k}^{n}=\left\{\begin{array}{cc}
1-\frac{v_{2}}{v_{1}+v_{2}} e^{v_{1} \mathcal{T}_{R, k}}, & \mathcal{T}_{R, k} \leq 0 \\
\frac{v_{1}}{v_{1}+v_{2}} e^{-v_{2}} \mathcal{T}_{R, k}, & \mathcal{T}_{R, k}>0
\end{array} .\right.
$$

APPENDIX B: DERIVATION OF THE PEP $\operatorname{Pr}\left(\tilde{u}_{k} \mid u_{k}\right)$ AT THE CRBS

The pairwise probabilities $\operatorname{Pr}\left(\tilde{u}_{k} \mid u_{k}\right)$ of the SU-to-CRBS link can be derived as

$$
\operatorname{Pr}\left(\tilde{u}_{k}=1 \mid u_{k}\right)=\sum_{\overline{\boldsymbol{u}}_{k}} \operatorname{Pr}\left(\tilde{u}_{k}=1 \mid u_{k}, \overline{\boldsymbol{u}}_{k}\right) \operatorname{Pr}\left(\overline{\boldsymbol{u}}_{k} \mid u_{k}\right)
$$

where

$$
\begin{aligned}
\operatorname{Pr}\left(\tilde{u}_{k}=1 \mid u_{k}, \overline{\boldsymbol{u}}_{k}\right) & =\operatorname{Pr}\left(\Psi_{k} \geq \mathcal{T}_{C, k} \mid u_{k}, \overline{\boldsymbol{u}}_{k}\right) \\
& =\int_{\mathcal{T}_{C, k}}^{\infty} f_{\Psi_{k}}\left(\Psi_{k} \mid u_{k}, \overline{\boldsymbol{u}}_{k}\right) d \Psi_{k} .
\end{aligned}
$$

The PDF $f_{\Psi_{k}}\left(\Psi_{k} \mid u_{k}, \overline{\boldsymbol{u}}_{k}\right)$ can be evaluated by noting that the characteristic function of $\Psi_{k} \mid u_{k}, \overline{\boldsymbol{u}}_{k}$ is given by

$$
\phi_{\Psi_{k}}\left(s \mid \overline{\boldsymbol{u}}_{k}, u_{k}\right)=\phi_{y_{k}^{0}}\left(s \mid u_{k}\right) \prod_{n=1}^{N} \phi_{\varphi_{k}^{n}}\left(s \mid u_{k}^{n}, u_{k}\right) .
$$

The first term in (32) $u_{k}$ can be directly obtained as

$$
\phi_{y_{k}^{0}}\left(s \mid u_{k}\right)=\frac{-v_{1}^{0} v_{2}^{0}}{\left(s-v_{2}^{0}\right)\left(s+v_{1}^{0}\right)}
$$

and $\phi_{-\varphi_{k}^{n}}\left(s \mid \hat{u}_{k}^{n}\right)$ can be evaluated as

$$
\begin{aligned}
\phi_{\varphi_{k}^{n}}\left(s \mid \hat{u}_{k}^{n}\right) & =E\left[e^{-s \varphi_{k}^{n}}\right] \\
& =\int_{-\infty}^{c_{1}} e^{-s d_{k}} f\left(y_{k}^{n} \mid \hat{u}_{k}^{n}\right) d y_{k}^{n} \\
& +\int_{c_{1}}^{c_{2}} e^{-s\left(g_{k} y_{k}+l_{k}\right)} f\left(y_{k}^{n} \mid \hat{u}_{k}^{n}\right) d y_{k}^{n} \\
& +\int_{c_{2}}^{\infty} e^{-s b_{k}} f\left(y_{k}^{n} \mid \hat{u}_{k}^{n}\right) d y_{k}^{n}
\end{aligned}
$$

where $f\left(y_{k}^{n} \mid \hat{u}_{k}^{n}\right)$ is given by

$$
f\left(y_{k}^{n} \mid \hat{u}_{k}^{n}\right)=\frac{v_{1}^{n} v_{2}^{n}}{v_{1}^{n}+v_{2}^{n}}\left(e^{v_{2}^{n} y_{k}^{n}} \Phi\left(-y_{k}^{n}\right)+e^{-v_{1}^{n} y_{k}^{n}} \Phi\left(y_{k}^{n}\right)\right)
$$

and $v_{i}^{n} \forall i \in\{1,2\}$ can be expressed as

$$
v_{i}^{n}=\sqrt{\left(w_{k}^{n}\right)^{2}+\frac{1}{\left(C_{k}^{n}\right)^{2}\left(\sigma_{r_{k}^{n}}^{2} \sigma_{\beta_{k}}^{2}-\left|\mu_{r_{k}^{n}, \beta_{k}^{n}}\right|^{2}\right)}}-(-1)^{i} w_{k}^{n} .
$$

The parameters required to compute $v_{i}^{n}$ are defined as

$$
\begin{aligned}
w_{k}^{n} & =\frac{\mu_{r_{k}^{n}, \beta_{k}^{n}}}{C_{k}^{n}\left(\sigma_{r_{k}^{n}}^{2} \sigma_{\beta_{k}}^{2}-\left|\mu_{r_{k}^{n}, \beta_{k}^{n}}\right|^{2}\right)}, C_{k}^{n}=\frac{2}{\sigma_{\xi_{k}}^{2}} \sqrt{\mathcal{P}_{k}^{n}}, \\
\mu_{r_{k}^{n}, \beta_{k}^{n}} & =\sqrt{\mathcal{P}_{k}^{n}} \sigma_{\beta_{k}}^{2} \hat{u}_{k}^{n}, \sigma_{r_{k}^{n}}^{2}=\mathcal{P}_{k}^{n} \sigma_{\beta_{k}}^{2}+\sigma_{\xi_{k}}^{2} .
\end{aligned}
$$

Consequently, the integral in (35) can be evaluated as

$$
\begin{aligned}
& \phi_{\varphi_{k}^{n}}\left(s \mid \hat{u}_{k}^{n}\right)=\frac{1}{v_{1}^{n}+v_{2}^{n}}\left(v_{1}^{n} e^{\left(-s d_{k}+v_{2}^{n} c_{1}\right)}+v_{2}^{n} e^{-\left(s b_{k}+v_{1}^{n} c_{2}\right)}\right. \\
& \left.+v_{1}^{n} v_{2}^{n} e^{-s l_{k}}\left(\frac{1-e^{\left(-s g_{k}+v_{2}^{n}\right) c_{1}}}{-s g_{k}+v_{2}^{n}}+\frac{e^{\left(-s g_{k}-v_{1}^{n}\right) c_{2}}-1}{-s g_{k}-v_{1}^{n}}\right)\right) .
\end{aligned}
$$

Thus, the PDF $f_{\Psi_{k}}\left(\Psi_{k}\right)$ is evaluated by computing the inverse Laplace transform of $\phi_{\Psi_{k}}\left(s \mid \overline{\boldsymbol{u}}_{k}, u_{k}\right)$; however, the number of terms in (32) increases substantially with the number of relays, and hence, the single relay case is considered. For multiple relays, numerical methods such as the Gauss-Chebyshev 
TABLE I

The Relative PSSE using The ADAPtive Threshold $\tau_{V}$ AND THe StatiC Threshold $\tau_{0}$ Where $K=8, P_{f a}=0.05, N=1$, SNR=5 $d B$.

\begin{tabular}{|c|c|c|c|c|c|c|c|c|c|c|c|}
\hline & \multicolumn{10}{|c|}{$P^{\mathrm{d}}$} \\
\hline & & 0.5 & 0.55 & 0.6 & 0.65 & 0.7 & 0.75 & 0.8 & 0.85 & 0.9 & 0.95 \\
\hline \multirow{10}{*}{$\operatorname{Pr}\left(H_{1}\right)$} & 0.01 & 5.5 & 5.6 & 5.3 & 6.1 & 6.9 & 7.9 & 9.0 & 1.2 & 1.4 & 1.9 \\
\hline & 0.11 & 2.9 & 3.2 & 1.1 & 1.3 & 1.7 & 2.4 & 3.3 & 1.0 & 1.0 & 1.0 \\
\hline & 0.21 & 2.2 & 2.5 & 1.0 & 1.1 & 1.5 & 2.1 & 2.8 & 1.0 & 1.0 & 1.6 \\
\hline & 0.31 & 1.8 & 2.0 & 1.0 & 1.0 & 1.4 & 1.8 & 2.3 & 1.0 & 1.0 & 2.0 \\
\hline & 0.41 & 1.6 & 1.8 & 1.0 & 1.0 & 1.2 & 1.6 & 1.8 & 1.0 & 1.1 & 1.6 \\
\hline & 0.51 & 1.4 & 1.5 & 1.0 & 1.0 & 1.1 & 1.3 & 1.4 & 1.0 & 1.0 & 1.0 \\
\hline & 0.61 & 1.2 & 1.3 & 1.0 & 1.0 & 1.1 & 1.1 & 1.0 & 1.0 & 1.0 & 1.0 \\
\hline & 0.71 & 1.1 & 1.2 & 1.0 & 1.0 & 1.0 & 1.0 & 1.0 & 1.0 & 1.0 & 1.0 \\
\hline & 0.81 & 1.0 & 1.1 & 1.0 & 1.0 & 1.0 & 1.0 & 1.0 & 1.0 & 1.0 & 1.0 \\
\hline & 0.91 & 1.0 & 1.0 & 1.1 & 1.2 & 1.1 & 1.0 & 1.0 & 1.1 & 1.0 & 1.0 \\
\hline
\end{tabular}

quadrature rules can be used to evaluate the integral. For the where

single relay case $f_{\Psi_{k}}\left(\Psi_{k}\right)$ can be written as

$$
\begin{aligned}
f_{\Psi_{k}}\left(\Psi_{k}\right) & =\frac{v_{1}^{0} v_{2}^{0} v_{1}^{1} v_{2}^{1}}{\left(v_{1}^{1}+v_{2}^{1}\right)\left(v_{1}^{0}+v_{2}^{0}\right)}\left(e^{v_{2}^{1} c_{1}}\left[\frac{1}{v_{2}^{1}} T_{1}-T_{3}\right]+T_{2}\right. \\
& \left.-e^{-v_{1}^{1} c_{2}}\left[T_{4}-\frac{1}{v_{1}^{1}} T_{6}\right]+T_{5}\right)
\end{aligned}
$$

where

$$
\begin{aligned}
T_{1}= & e^{v_{2}^{0}\left(\Psi_{k}-d_{k}\right)} \Phi\left(-\Psi_{k}+d_{k}\right)+e^{-v_{1}^{0}\left(\Psi_{k}-d_{k}\right)} \Phi\left(\Psi_{k}-d_{k}\right) \\
T_{2}= & A_{2}\left(B_{2} e^{\frac{v_{2}^{1}}{g_{k}}\left(\Psi_{k}-l_{k}\right)}+e^{v_{2}^{0}\left(\Psi_{k}-l_{k}\right)}\right) \Phi\left(-\Psi_{k}+l_{k}\right) \\
+ & C_{2} e^{-v_{1}^{0}\left(\Psi_{k}-l_{k}\right)} \Phi\left(\Psi_{k}-l_{k}\right) \\
T_{3}= & A_{3}\left(B_{3} e^{\frac{v_{2}^{1}}{g_{k}}\left(\Psi_{k}-g_{k} c_{1}-l_{k}\right)}+e^{v_{2}^{0}\left(\Psi_{k}-g_{k} c_{1}-l_{k}\right)}\right) \\
& \times \Phi\left(-\Psi_{k}+g_{k} c_{1}+l_{k}\right) \\
& +C_{3} e^{-v_{1}^{0}\left(\Psi_{k}-g_{k} c_{1}-l_{k}\right)} \Phi\left(\Psi_{k}-g_{k} c_{1}-l_{k}\right) \\
T_{4}= & -A_{4}\left(B_{4} e^{-\frac{v_{1}^{1}}{g_{k}}\left(\Psi_{k}-g_{k} c_{2}-l_{k}\right)}+e^{-v_{1}^{0}\left(\Psi_{k}-g_{k} c_{2}-l_{k}\right)}\right) \\
\times & \Phi\left(\Psi_{k}-g_{k} c_{2}-l_{k}\right) \\
+ & C_{4} e^{v_{2}^{0}\left(\Psi_{k}-g_{k} c_{2}-l_{k}\right)} \Phi\left(-\Psi_{k}+g_{k} c_{2}+l_{k}\right) \\
T_{5}\left(\Psi_{k}\right)= & -A_{5}\left(B_{5} e^{-v_{1}^{1} / g_{k}\left(\Psi_{k}-l_{k}\right)}+e^{-v_{1}^{0}\left(\Psi_{k}-l_{k}\right)}\right) \\
& \times \Phi\left(\Psi_{k}-l_{k}\right)+C_{5} e^{v_{2}^{0}\left(\Psi_{k}-l_{k}\right)} \Phi\left(-\Psi_{k}+l_{k}\right) \\
T_{6}\left(\Psi_{k}\right)= & e^{v_{2}^{0}\left(\Psi_{k}-b_{k}\right)} \Phi\left(b_{k}-\Psi_{k}\right)+e^{-v_{1}^{0}\left(\Psi_{k}-b_{k}\right)} \Phi\left(\Psi_{k}-b_{k}\right)
\end{aligned}
$$

$$
\begin{aligned}
& A_{i}=\frac{1}{\left[\frac{v_{\iota}^{1}}{g_{k}}-v_{\iota}^{0}\right] g_{k}}, B_{i}=\frac{-\left(v_{1}^{0}+v_{2}^{0}\right)}{\frac{v_{\iota}^{1}}{g_{k}}+v_{2 / \iota}^{0}}, \\
& C_{i}=\frac{1}{\left(v_{2 / \iota}^{0} g_{k}+v_{\iota}^{1}\right)}, \iota=\lfloor\sqrt[i]{3 i}\rfloor .
\end{aligned}
$$

Consequently, evaluating the integral in (31) yields

$$
\begin{aligned}
& \operatorname{Pr}\left(\tilde{u}_{k}=1 \mid u_{k}, \overline{\boldsymbol{u}}_{k}\right)=\frac{v_{1}^{0} v_{2}^{0} v_{1}^{1} v_{2}^{1}}{\left(v_{1}^{1}+v_{2}^{1}\right)\left(v_{1}^{0}+v_{2}^{0}\right)}\left(e^{v_{2}^{1} c_{1}}\right. \\
&\left.\quad \times\left[\frac{1}{v_{2}^{1}} I_{1}-I_{3}\right]+I_{2}-e^{-v_{1}^{1} c_{2}}\left[I_{4}-\frac{1}{v_{1}^{1}} I_{6}\right]+I_{5}\right)
\end{aligned}
$$

and the terms $I_{1}$ through $I_{6}$ are given in (49) through (54), respectively. Similarly, $\operatorname{Pr}\left(\tilde{u}_{k}=-1 \mid u_{k}, \overline{\boldsymbol{u}}_{k}\right)$ can be expressed as described in (48) where $I_{1}, I_{2}, \ldots, I_{6}$ are given in (55) through (60), respectively.

\section{REFERENCES}

[1] M. Kalil, A. Shami, A. Al-Dweik, and S. Muhaidat, "Low-complexity power-efficient schedulers for LTE uplink with delay-sensitive traffic," IEEE Trans. Veh. Technol., vol. 64, no. 10, pp. 4551-4564, Oct. 2015.

[2] A. Das, F. Catthoor, A. Bourdoux and B. Gyselinckx, "Energy efficient mapping of LTE-A PHY signal processing tasks on microservers," IEEE Trans. Green Commun. Netw., IEEE early access, doi: 10.1109/TGCN.2018.2794477.

[3] Future Mobile Data Usage and Traffic Growth, Ericsson, available online at https://www.ericsson.com/en/mobility-report/future-mobile-datausage-and-traffic-growth.

[4] I. Akyildiz, W. Lee, M. Vuran, and S. Mohanty "A survey on spectrum management in cognitive radio networks," IEEE Commun. Mag., vol. 46 , no. 4, pp. 40-48, Feb. 2008

[5] W. Lee, and I. Akyildiz, "Optimal spectrum sensing framework for cognitive radio Networks," IEEE Trans. Wireless Commun., vol. 7, no. 10, pp. 3845-3857, Oct. 2008.

[6] A. Ali and W. Hamouda, "Advances on spectrum sensing for cognitive radio networks: theory and applications," IEEE Commun. Surveys Tuts., vol. 11, no. 2, pp. 1277-1304, Nov. 2016

[7] Z. Qin, J. Wang, J. Chen, and L. Wang, "Adaptive compressed spectrum sensing based on cross validation in wideband cognitive radio system," IEEE Syst. J., vol. 11, no. 4, pp. 2422-2431, Dec. 2017.

[8] Y. He, J. Xue, T. Ratnarajah, M. Sellathurai, and F. Khan, "On the performance of cooperative spectrum sensing in random cognitive radio networks," IEEE Syst. J., vol. 12, no. 1, pp. 881-892, Mar. 2018. 
[9] S. Atapattu, C. Tellambura, and H. Jiang, "Energy detection based cooperative spectrum sensing in cognitive radio networks," IEEE Trans. Wireless Commun., vol. 10, no. 4, pp. 1232-1241, Apr. 2011.

[10] L. Khalid and A. Anpalagan, "Reliability-based decision fusion scheme for cooperative spectrum sensing," IET Commun., vol. 8, no. 14, pp. 2423-2432, Sep. 2014

[11] K. Cichon, A. Kliks, and H. Bogucka, "Energy-efficient cooperative spectrum sensing: a survey," IEEE Commun. Surveys Tuts., vol. 18, no. 3, pp. 1861-1886, 2016.

[12] H. Chen, M. Zhou, L. Xie, and J. Li, "Cooperative spectrum sensing with M-ary quantized data in cognitive radio networks under SSDF attacks," IEEE Trans. Wireless Commun., vol. 16, no. 8, pp. 5244-5257, Aug. 2017.

[13] R. Niu, B. Chen, and P. Varshney, "Fusion of decisions transmitted over Rayleigh fading channels in wireless sensor networks," IEEE Trans. Signal Process., vol. 54, no. 3, pp. 1018-1027, Mar. 2006.

[14] A. Lei and R. Schober, "Coherent max-log decision fusion in wireless sensor networks," IEEE Trans. Commun., vol. 58, no. 5, pp. 1327-1332, May 2010.

[15] D. Ciuonzo, G. Romano, and P. Salvo Rossi, "Channel-aware decision fusion in distributed MIMO wireless sensor networks: decode-and-fuse vs. decode-then-fuse," IEEE Trans. Wireless Commun., vol. 11, no. 8, pp. 2976-2985, Aug. 2012.

[16] H. Ahmadi and A. Vosoughi, "Impact of wireless channel uncertainty upon distributed detection systems," IEEE Trans. Wireless Commun., vol. 12 , no. 6 , pp. 2566-2577, Jun. 2013

[17] M. Al-Jarrah, N. Al-Ababneh, M. Al-Ibrahim, and R. Al-Jarrah, "Cooperative OFDM for semi distributed detection in wireless sensor networks," Int. J. Electron. Commun., vol. 68, no. 10, pp.1022-1029, Oct. 2014.

[18] M. Al-Jarrah, R. Al-Jarrah, and N. Al-Ababneh, "Decision fusion in mobile wireless sensor networks using cooperative multiple symbol differential space time coding," Int. J. Electron. Commun.,vol.80, pp.127136, Oct. 2017.

[19] M. Al-Jarrah, A. Al-Dweik, M. Kalil and S. Ikki, "Efficient decision fusion for cooperative wireless sensor networks," International Conference on Electrical and Computing Technologies and Applications (ICECTA), Ras Al Khaimah, 2017, pp. 1-5.

[20] M. Al-Jarrah, A. Al-Dweik, M. Kalil and S. Ikki, "Decision fusion in distributed cooperative wireless sensor networks," IEEE Trans. Veh. Technol, Early Access.
[21] A. Paul, A. Daniel, A. Ahmad, and S. Rho, "Cooperative cognitive intelligence for internet of vehicles," IEEE Syst. J., vol. 11, no. 3, pp. 1249-1258, Sep. 2017.

[22] P. Salvo Rossi, D. Ciuonzo, and G. Romano, "Orthogonality and cooperation in collaborative spectrum sensing through MIMO decision Fusion," IEEE Trans. Wireless Commun., vol. 12, no. 11, pp. 5826-5836, Nov. 2013.

[23] A Patel, H. Ram, A. Jagannatham, and P. Varshney, "Robust cooperative spectrum sensing for MIMO cognitive radio networks under CSI uncertainty," IEEE Trans. Signal Process., vol. 66, no. 1, pp. 18-33, Jan. 2018.

[24] M. Azmi and H. Leib, "Coded collaborative spectrum sensing with joint channel decoding and decision fusion," IEEE Trans. Wireless Commun., vol. 14, no. 4, pp. 2017-2031, Apr. 2015

[25] M. Azmi and H. Leib, "Multi-channel cooperative spectrum sensing that integrates channel decoding with fusion based decision," IEEE Trans. Aerosp. Electron. Syst., IEEE early access, doi: 10.1109/TAES.2018.2807300.

[26] D. Das and S. Das, "A survey on spectrum occupancy measurement for cognitive radio," Wireless Pers. Commun., vol. 85, no. 4, pp. 2581-2598, Dec. 2015

[27] Y. Chen and H. Oh, "A survey of measurement-based spectrum occupancy modeling for cognitive radios," IEEE Commun. Surveys Tuts., vol. 18 , no. 1, pp. 848-859, 1st Quart., 2016.

[28] M. Aslam and M. Ayyaz, "Real-time delivery of 4G services with crosslayered and power-optimized cognitive radio architecture," IEEE Syst. $J$., vol. 10, no. 1, pp. 325-334, Mar. 2016.

[29] R. Mesleh and S. Ikki, "Performance analysis of spatial modulation with multiple decode and forward relays," IEEE Wireless Commun. Lett., vol. 2, no. 4, pp. 423-426, Aug. 2013.

[30] S. Ikki and M. Ahmed, "Performance analysis of adaptive decode-andforward cooperative diversity networks with best-relay selection," IEEE Trans. Commun., vol. 58, no. 1, pp. 68-72, Jan. 2010.

[31] D. Duan, L. Scharf, and L. Yang, "LD approach to asymptotically optimum sensor fusion," IET Commun., vol. 11, no. 5, pp. 680-686, Apr. 2017.

[32] M. Fernandez and S. Williams, "Closed-form expression for the Poissonbinomial probability density function," IEEE Trans. Aerosp. Electron. Syst., vol. 46, no. 2, pp. 803-817, Apr. 2010. 